\title{
The Effect of Berberine on the Transcriptome and Proteome of $E$. coli
}

\author{
Zhang Shilei ${ }^{1,2, \# a}$, Jia Ze ${ }^{2, \# b}$, Zhai Xianghe2,\#b, Wang Chunguang,,\#b , Zhang Tie 2,\#b*
}

${ }^{1}$ College of Animal Science and Technology, Agricultural University of Hebei, Baoding 071001, China

${ }^{2}$ College of Veterinary Medical \& Traditional Chinese Veterinary College, Agricultural University of Hebei, Baoding 071001, China

*Corresponding Author: Zhang Tie

E-mail: zhangtie1998@163.com(ZT)

This study was conducted at Hebei Agricultural University.

The procedures in this study were approved by the Institutional Animal Care and Ethics Committee of Agricultural University of Hebei (approval no. IACECHEBAU20150315).

This study was financially supported by the National Natural Science Foundation of China (No. 31572560); the Hebei Key Technology R\&D Program grant (No. 16222701D) from the Department of Science \& Technology, Hebei Province, China. We are grateful to Li Yan for editing this article. 


\begin{abstract}
Berberine is commonly used to treat diarrhea in China, and the antibacterial properties of berberine have been confirmed. High-throughput sequencing technology was used to explore the changes induced in $E$. coli by berberine. After treatment with berberine, the expression of RstA and YbjG were found to be significantly different by RNA-seq and quantitative proteomics. However, the levels of MdtA, PmrA, LolD, LptG, MlaB, RcsF, and DppB were found to be significantly different by quantitative proteomics. Transcriptome sequencing did not yield as many results as proteome sequencing. The results of small RNA prediction showed increased sRNA00002 levels. The study showed that the differentially expressed mRNAs and proteins were associated with multidrug-resistant efflux systems. It can be inferred that berberine reduces $E$. coli antibiotic resistance. The results of this study are undoubtedly valuable to other researchers.
\end{abstract}

Keywords: berberine; E. coli; RNA-seq; proteomics; antibiotics

\title{
1 Introduction
}

Berberine, an alkaloid originally extracted from Coptis chinensis and other herb plants, significantly inhibits the growth of bacteria, viruses and protozoa ${ }^{1,2}$. Researchers have found that enhancement of the fluconazole-susceptibility of Candida tropicalis is mediated by berberine ${ }^{3}$. Wang Jie et al. determined the minimum inhibitory concentrations (MIC) of Belamcanda chinensis, C. chinensis Franch, and the Sanhuang decoction (Scutellaria baicalensis Georgi, C. chinensis Franch, Rheum palmatum L.) against multidrug-resistant Acinetobacter baumannii, and the results demonstrated that the reversal rates were $4.2 \%, 2.8 \%$ and $11.1 \%$, respectively ${ }^{4}$. Eumkeb $\mathrm{G}$. et al. claimed that galangin, quercetin and baicalein all had the potential to eliminate the tolerance of penicillin-resistant Staphylococcus aureus to AMP antibiotics ${ }^{5}$. Bouhdid S. et al. discovered that thymol and carvacrol in 
oregano are involved in the regulation of membrane permeability in bacteria and function by destroying the cell membrane structure and increasing sensitivity of multidrug-resistant bacteria to antibiotics ${ }^{6}$. Other studies have proven that baicalin can inhibit bacterial membrane systems ${ }^{7}$. When penicillinresistant $S$. aureus was treated with chlorogenic acid, the cell membrane composition of the bacteria changed, and the susceptibility of $S$. aureus to penicillin improved ${ }^{8}$. Modern pharmacologists have shown that bacteria become sensitive to a variety of antibiotics when treated with berberine. Berberine can inhibit the function of bacterial cell membranes ${ }^{9}$, reduce the lipopolysaccharide content of bacterial cell walls, and increase the susceptibility of bacteria to antibiotics ${ }^{10,11}$. In addition, the glycolic acid oxidation and pyruvate oxidation pathways are inhibited by berberine during the process of glucose metabolism ${ }^{12}$. However, the mechanism via which berberine mediates the enhancement of the antibiotic-susceptibility of Escherichia coli remains unclear.

High-throughput sequencing technology is widely used in scientific research. However, there remains a gap between traditional Chinese medicinal research and high-throughput sequencing technology. There has been no research to identify the role of berberine in E. coli via transcriptome and proteome analyses. To explore the changes mediated by berberine in E. coli, RNA-seq and quantitative proteomic techniques were used in this study. We were able to obtain complete transcriptomic and proteomic data for $E$. coli with or without berberine treatment. In this study, we found that the expression of RstA (b1608) and YbjG (b0841) was significantly different by RNA-seq and quantitative proteomic analyses. However, the expression of MdtA (b2074), PmrA (b4113), LolD (b1117), LptG (b4262), MlaB (b3191), $R c s F$ (b0196), and $D p p B$ (b3543) was significantly different by quantitative proteomic analysis. The results of small RNA prediction showed that the level of sRNA00002 increases. Gene Ontology (GO) and Kyoto Encyclopedia of Genes and Genomes (KEGG) enrichment analyses showed that these significantly differentially expressed genes (DEGs) were primarily annotated as being involved in cell 
surface signaling, ion binding, and lipid metabolism, while the differentially expresses proteins were annotated as membrane proteins, cell wall synthesis proteins and cell membrane synthesis proteins.

\section{Materials and Methods}

A strain of E. coli used in this study was isolated from the Animal Hospital of Hebei Agricultural University, Baoding.

\subsection{RNA-seq}

RNA degradation and contamination were monitored by agarose gel electrophoresis, and purity was checked by using a Nano Photometer spectrophotometer (Nano drop 2000, Roche, Switzerland), while concentration was measured by using a Qubit RNA Assay Kit (Thermo, US) with a Qubit 2.0 fluorometer. Then, RNA integrity (Life Technologies, CA) was assessed by an Agilent Bioanalyzer 2100 system (Agilent, US). After passing the test, sequencing libraries were generated by the NEBNext Ultra Directional RNA Library Prep Kit (Biolabs, UK), after which the library fragments were purified with an AMPure XP system (Beckman, US). In addition, library quality was assessed on an Agilent Bioanalyzer 2100 system. After inspection, the library was pooled for Illumina HiSeqTM 2500 sequencing (Illumia, US).

\subsubsection{Mapping reads to the reference genome}

Reference genome and gene model annotation files were downloaded directly from the genome website. Bowtie2-2.2.3 was used to both building an index of the reference genome and align clean reads to the reference genome. In this study, the reference genome selected for use was that of E. coli str. K-12 substr. mg1655 (ftp://ftp.ensemblgenomes.org/pub/bacteria/release

31/fasta/bacteria_0_collection/escherichia_coli_str_k_12_substr_mg1655/dna/).

\subsubsection{Small RNA prediction and changes in expression}


Non-coding RNAs with lengths of 50 to $500 \mathrm{nt}$ are generally defined as small RNA. New intergenic region transcripts were found by using Rockhopper software. The newly predicted transcribed region was annotated by the BLASTx and the non-redundant (NR) nucleotide library. Non-commented transcripts were non-coding for small RNA. Secondary structure prediction and target gene prediction of the candidate small RNA were carried out by RNAfold software and IntaRNA software, respectively.

\subsubsection{Quantitative analysis of gene expression levels}

Fragments per kilobase of exon per million fragments mapped (FPKM), which considers the effect of sequencing depth and gene length for read counts at the same time, is currently the most commonly used method for estimating gene expression levels. HTSeq v0.6.1 was used to count the number of reads mapped to each gene. The FPKM of each gene was calculated based on the length of the gene and read counts mapped to the gene. Differentially expressed gene sequencing (DEGseq) analysis of the two conditions was performed by the DEGseq R package. The threshold for significantly different expression was a P-value less than 0.005 and 2-fold change (FC) in threshold conditions $(\log 2(\mathrm{FC})>1)$.

\subsubsection{Gene Ontology (GO) and Kyoto Encyclopedia of Genes and Genomes (KEGG) enrichment analyses of differentially expressed genes (DEGs)}

GO enrichment analysis of differentially expressed genes was implemented by the GOseq R package, in which gene length bias was corrected. GO terms with corrected P-values less than 0.05 were considered significantly enriched with differentially expressed genes.

KEGG is a database resource for understanding high-level functions and utilities of biological systems, such as cells, organisms and ecosystems, from molecular-level information, especially from large-scale molecular datasets generated by genome sequencing and other high-throughput experimental technologies (http://www.genome.jp/kegg/). We used KOBAS software to test the statistical enrichment of differentially expressed genes in KEGG pathways. 


\subsubsection{Fluorescence-based real-time quantitative PCR (RT-qPCR) for validation of DEGs}

To verify the reliability of RNA-seq results, 8 differentially expressed genes were selected for RTqPCR validation (Table 1).

Table 1 Primer sequences used for RT-qPCR

\subsection{Quantitative proteomic sequencing}

The total proteins were analyzed by the Bradford protein assay and SDS-PAGE. Mass spectrometry was performed after passing the test assay. The proteins were subjected to capillary high-performance liquid chromatography (HPLC) on an Easy-nLC 1000 instrument after reductive alkylation and trypsin hydrolysis and then subjected to mass spectrometry using a Q Exactive HF mass spectrometer.

\subsubsection{Mass spectrometric analysis and identification of differentially expressed proteins}

This study used the UniProt Escherichia coli database. The original data were analyzed by Mascot and Proteome Discoverer software, while Proteome Discover 2.0 software combined with Mascot's algorithm was used for qualitative and quantitative calculation of proteomics data. The extracted proteins were analyzed by mass spectrometry, and the results were filtered with a peptide false discovery rate (FDR) less than 0.01 . The threshold criterion for significant differences in the ratios of the protein levels identified was $1.5 \mathrm{FC}(\mathrm{FC} \geq 1.5$ is upregulated; $\mathrm{FC} \leq 0.667$ is downregulated; $0.667<\mathrm{FC}<1.5$ implies no significant change).

\subsubsection{GO and KEGG enrichment analyses of differentially expressed proteins}

Differentially expressed proteins were analyzed by GO functional annotation analysis and KEGG pathway annotation analysis to identify significantly enriched GO functions and KEGG pathways. Terms with P-values less than 0.05 were considered significantly enriched with differentially expressed genes. 


\section{Results}

\subsection{Isolation, Identification and Screening of $E$. coli}

Coli0 was identified as $E$. coli. To investigate the effect of berberine, E. coli was treated with berberine at $250 \mu \mathrm{g} / \mathrm{mL}$ (named Coli1). RNA and protein were extracted for RNA-seq and quantitative proteomic studies.

\subsection{RNA-seq}

To determine the effect of berberine on bacterial mRNA, we performed transcriptase sequencing. By RNA-seq, 14.51 million and 13.71 million clean reads were obtained for Coli0 and Coli1, respectively, and 92.18\%, 93.97\% were mapped to the reference genomes of Coli0 and Coli1, respectively. The Q20 and Q30 of Coli0 and Coli1 were 98.84\%, 98.76\% and 96.82\%, 96.63\%, respectively. There were 45 DEGs between Coli0 and Coli1. Among these DEGs, 30 genes were upregulated, and 15 genes were downregulated (Supporting Table 1, Fig 1).

Fig 1 Volcano plot of DEGs between Coli1 and Coli0

\subsubsection{Small RNA prediction}

New intergenic region transcripts were identified by comparison of the BLASTx and NR libraries. The newly predicted transcribed region was annotated, and the unlabeled transcripts were used as candidate non-coding small RNAs. Secondary structure prediction and target gene prediction of the candidate small RNAs were carried out by RNAfold software and IntaRNA software, respectively. A 72-bp small RNA was predicted, namely, sRNA00002 (Fig 2). The target gene of sRNA00002 is DppB; the complementary position is $849-903$; and the dissociation energy is $-13.3768 \mathrm{kcal} / \mathrm{mol}$. In berberinetreated E. coli, the expression of sRNA00002 increased from 619.74 FPKM to 664.15 FPKM. 
Fig 2 sRNA00002 secondary structure prediction

\subsubsection{Analysis of GO functions at the RNA level}

To investigate the biological processes associated with berberine-induced levofloxacin resistance in $E$. coli, we performed GO enrichment analysis on DEGs detected by RNA-seq. The enriched GO functions included cell surface receptor signaling pathway, lipid modification, positive regulation of immune system process, antigen receptor-mediated signaling pathway, regulation of antigen receptormediated signaling pathway, ion binding, Wnt receptor signaling pathway, cellular lipid metabolic process, lipid phosphorylation, regulation of signal transduction, regulation of cell communication, regulation of signaling, single-organism metabolic process, DNA polymerase III complex, DNA polymerase complex, lipid metabolic process, signal transduction, anion binding, signaling, and singleorganism signaling (Table 2).

Table 2 GO function enrichment at the RNA level in Coli0 and Coli1

\subsubsection{Analysis of KEGG pathways at the RNA level}

To study the metabolic pathways associated with levofloxacin resistance in E. coli, KOBAS software was utilized to detect metabolic pathways affected by the DEGs. Seven significant KEGG pathways were identified as being enriched, namely, fatty acid degradation, glutathione metabolism, fatty acid metabolism, peptidoglycan biosynthesis, microbial metabolism in diverse environments, metabolic pathways, and two-component system (Table 3).

Table 3 KEGG enrichment at the RNA level in Coli0 and Coli1 


\subsubsection{RT-qPCR}

Eight genes were randomly selected from 45 significantly differentially expressed genes for validation. Comparison of the sequencing results with the multiple expression values of each gene obtained by RT-qPCR demonstrated that the trend of gene expression remains roughly the same; however, there is a difference between the two trends. This discrepancy may be a result of the differences in instrumentation, reagents, operating errors, or algorithms (Fig 3).

Fig 3 Expression levels of eight genes from the Coli0 and Coli1 determined by RT-qPCR and RNA-seq. Positive values on the $\mathrm{Y}$ axis represent upregulation, and negative values represent downregulation.

\subsection{Quantitative proteomic sequencing}

To characterize the effect of berberine on bacterial protein synthesis, we used label-free proteomic sequencing analysis. A total of 2003 proteins were obtained from quantitative proteomic sequencing. There were 136 unique proteins in Coli0, 162 unique proteins in Coli1. There were 331 downregulated proteins and 291 upregulated proteins in Coli1. To study the changes in resistance-related proteins in thee mutant $E$. coli, we analyzed the differential expression of membrane proteins, cell wall synthesis proteins and cell membrane synthesis proteins.

\subsubsection{GO Analysis of antibiotic-resistant proteins}

In the GO function enrichment analysis, 37 terms were significantly enriched, including plasma membrane, response to metal ion, response to inorganic substance, metal ion transport, glycerol catabolic process, organic acid transmembrane transporter activity, carboxylic acid transmembrane transporter activity, ion transport, nitrogen compound transport, integral component of membrane, transporter activity, organic anion transmembrane transporter activity, anion transport, organic anion 
transport, alditol metabolic process, carboxylic acid transport, glycerol metabolic process, cell

periphery, substrate-specific transmembrane transporter activity, transmembrane transporter activity, intrinsic component of membrane, substrate-specific transporter activity, alditol catabolic process, cellular response to inorganic substance, transcription factor activity, sequence-specific DNA binding, nucleic acid binding transcription factor activity, plasma membrane part, secondary active transmembrane transporter activity, transport, membrane part, response to transition metal nanoparticle, phenol-containing compound metabolic process, cell projection organization, racemase and epimerase activity, acting on amino acids and derivatives, metal ion transmembrane transporter activity, amino acid transport, and heme binding (Table 4).

Table 4 GO function enrichment at the protein level in Coli0 and Coli1

\subsubsection{KEGG analysis of antibiotic-resistant proteins}

The enriched KEGG pathways included ABC transporters; alanine, aspartate and glutamate metabolism; quorum sensing; glycerophospholipid metabolism; butanoate metabolism; nitrotoluene degradation; beta-alanine metabolism; two-component system; glycerolipid metabolism; peptidoglycan biosynthesis; cationic antimicrobial peptide (CAMP) resistance; beta-lactam resistance; vancomycin resistance; lipopolysaccharide biosynthesis; metabolism of xenobiotics by cytochrome P450; drug metabolism cytochrome P450; streptomycin biosynthesis; bacterial secretion system (Table 5).

Table 5 KEGG enrichment at the protein level in Coli0 and Coli1

\section{Discussion}


The response regulator of $\operatorname{RstAB}($ RstA) was significantly downregulated. The RstAB system is a bacterial iron uptake system, and downregulation of this system may lead to inhibition of bacterial iron absorption. The RstAB system is associated with assistance to harsh environments ${ }^{13}$. However, the relationship between $R s t A B$ and antibiotic resistance in bacteria has not been proven. Considering the use of berberine, the significant reduction in RstA expression might be associated with the stimulation of berberine.

Activation of the capsule synthesis promoter $(R c s F)$ was significantly downregulated. $R c s F$ is involved in cell wall synthesis, and downregulation of $\operatorname{Rcs} F$ leads to the inhibition of cell wall synthesis.

Modification of the $R c s F$ protein may act as an activating signal, so the expression of $R c s F$ was not significantly different when measured by RNA-seq ${ }^{14,15}$. Undecaprenyl-diphosphatase (YbjG) was significantly downregulated. $Y b j G$ is essential for the synthesis of undecaprenyl phosphate, a C55 lipid carrier for cell wall synthesis ${ }^{16}$. In addition, $Y b j G$ is similar to the bacitracin-resistance protein $B c r C$ of Bacillus licheniformis ${ }^{17}$. Touzé et al. inhibited the phosphorylation of lipid A in bacterial cell walls by using bacitracin, suggesting that lipid A phosphorylation in bacterial cell walls is directly associated with undecaprenyl pyrophosphate synthesis ${ }^{18}$. Incomplete cell walls weaken the antibiotic resistance of E. coli. This study proved that berberine can significantly inhibit bacterial cell wall synthesis.

The multidrug resistance protein $(M d t A)$ was significantly downregulated. $M d t A$ can cause bacteria to become dormant and resume growth after the antibiotic is no longer present. Bacteria can escape killing by antibiotics via transient dormancy, which occurs only during bacterial proliferation. The principle behind this phenomenon is that in the presence of antibiotics, the bacterial cells themselves produces a toxin protein that allows the bacteria to enter a dormant state from a growing state to achieve the goal of resisting antibiotics ${ }^{19}$. 
The transcriptional regulator of the multidrug resistance efflux pump $\operatorname{Pmr} A B(\operatorname{Pmr} A)$ was significantly downregulated. $P m r A B$ is the regulator of lipopolysaccharide (LPS) modification in E. coli, and PmrA is the transcriptional regulator of this system ${ }^{20}$. The expression of $P m r A B$ reduces the negative charge on the bacterial outer membrane, which affects the binding of bacteria to cationic antibiotics, leading to drug resistance ${ }^{21}$. Arroyo L. A. found that the change in PmrAB expression was associated with increased polymyxin resistance ${ }^{22}$. Interestingly, a decrease in PmrA expression attenuates the efflux of all drugs from E. coli, not only levofloxacin.

In this experiment, the expression levels of the ATP-binding protein of the lipoprotein release system (LolD) and permeation-associated enzyme of the lipopolysaccharide output system $(L p t G)$ were significantly decreased and that of the binding protein of phospholipid ABC transporter (MlaB) was significantly decreased. LolD provides energy for the transport of lipoproteins, and the absence of LolD will decrease the rate of transport of lipoproteins to the outer membrane ${ }^{23}$. Mla is involved in maintaining outer membrane lipid asymmetry via the transport of aberrantly localized phospholipids from the outer membrane to the inner membrane, while MlaB plays critical roles in both the assembly and activity of the transporter ${ }^{24}$. In gram-negative bacteria, lipid asymmetry is critical to the function of the outer membrane as a selectively permeable barrier. LPS can act against many antimicrobials. To assemble LPS on their surfaces, bacteria must extract LPS from the inner membrane to the outer membrane. The Lpt system transports LPS by using the ATPase LptB and the transmembrane domains $L p t F G^{25}$. Decreased $L l o D, L p t G$ and $M l a B$ expression will destroy cell membrane stability and weaken the role of the cell membrane barrier.

The expression of dipeptide $\mathrm{ABC}$ transporter permease $(D p p B)$ was significantly reduced. Peptide transporters play important roles in bacterial growth, not only in the supply of nutrients to bacteria but also in many processes of the bacterial lifecycle, such as signal transduction and chemotaxis ${ }^{26,27}$. There 
have been no studies to prove that the peptide transport system is associated with antibiotic resistance.

The small RNA that regulates $D p p B$ was also identified for the first time. We have not yet studied the peptide transport system further, but this system is a promising candidate as a system that is involved in E. coli antibiotic resistance. The newly discovered small RNA might be able to explain this phenomenon.

\section{Supplementary Material}

Supplementary Table Differentially expressed genes from Coli0 and Coli1. Between Coli0 and Coli1, the two E. coli cultures, a total of 45 genes were differentially expressed; "readcount" represents the relative expression level (FPKM); "log2 Fold change" represents the multiple of differential expression; negative values represent downregulates genes; positive values represent upregulated genes; "pvalue" and "qvalue" less than 0.01 represent significant differences. 


\section{References}

1. Mcdevitt JT. Berberine alkaloids as a treatment for chronic protozoally induced diarrhea. In: US; 2001.

2. Wang S, Yu J, Suvira M, et al. Uptake of and Resistance to the Antibiotic Berberine by Individual Dormant, Germinating and OutgrowingBacillusSpores as Monitored by Laser Tweezers Raman Spectroscopy. PloS one 2015;10:e0144183.

3. Shi G, Shao J, Wang T, et al. Mechanism of berberine-mediated fluconazole-susceptibility enhancement in clinical fluconazole-resistant Candida tropicalis isolates. Biomed Pharmacother 2017;93:709-712.

4. Wang J, Zhu JH, Zhang B, et al. Screening of traditional Chinese medicines that can reverse bacterial resistance. Chinese Pharmaceutical. 2014:1892-1896.

5. Eumkeb G, Sakdarat S, Siriwong S. Reversing beta-lactam antibiotic resistance of Staphylococcus aureus with galangin from Alpinia officinarum Hance and synergism with ceftazidime. Phytomedicine 2010;18:40-45.

6. Bouhdid S, Abrini J, Zhiri A, et al. Investigation of functional and morphological changes in Pseudomonas aeruginosa and Staphylococcus aureus cells induced by Origanum compactum essential oil. J Appl Microbiol 2009;106:1558-1568.

7. Kong JL, Chen YJ, Yan P, et al. Scutellariae decoction in vitro removal of biofilms of Pseudomonas aeruginosa in vitro study. Tuberculosis and Respiratory Diseases. 2006:347-348.

8. Zhang M.Antibacterial Effect of Traditional Chinese Medicine against Bacterial Drug Resistance. Western Medicine. 2013:122-124.

9. Xue DF, Zou ZY, Chen B, et al. Study on membrane injury mechanism of total alkaloids and berberine from Coptidis Rhizoma on Aeromonas hydrophila. Traditional Chinese Medicine. 2015;40:1787-1792.

10. Karaosmanoglu K, Sayar NA, Kurnaz IA, et al. Assessment of berberine as a multi-target antimicrobial: a multi-omics study for drug discovery and repositioning. OMICS 2014;18:42-53. 11. Antibacterial Mechanisms of Berberine and Reasons for Little Resistance of Bacteria. Chinese Herbal Medicines 2011:27-35.

12. Shirwaikar A, Shirwaikar A, Rajendran K, et al. In vitro antioxidant studies on the benzyl tetra isoquinoline alkaloid berberine. Biol Pharm Bull 2006;29:1906-1910.

13. Gao Q, Ye Z, Wang X, et al. RstA is required for the virulence of an avian pathogenic Escherichia coli $\mathrm{O} 2$ strain E058. Infection Genetics \& Evolution Journal of Molecular Epidemiology \& Evolutionary Genetics in Infectious Diseases 2015;29:180-188.

14. Rogov VV, Rogova NY, Bernhard F, et al. A disulfide bridge network within the soluble periplasmic domain determines structure and function of the outer membrane protein RCSF. The Journal of biological chemistry 2011;286:18775-18783.

15. Majdalani N, Heck M, Stout V, et al. Role of RcsF in signaling to the Rcs phosphorelay pathway in Escherichia coli. J Bacteriol 2005;187:6770-6778.

16. El Ghachi $M$, Derbise $A$, Bouhss $A$, et al. Identification of multiple genes encoding membrane proteins with undecaprenyl pyrophosphate phosphatase (UppP) activity in Escherichia coli. The Journal of biological chemistry 2005;280:18689-18695.

17. Harel YM, Bailone A, Bibi E. Resistance to bacitracin as modulated by an Escherichia coli homologue of the bacitracin $\mathrm{ABC}$ transporter BcrC subunit from Bacillus licheniformis. J Bacteriol $1999 ; 181: 6176-6178$. 
18. Touzé T, Tran AX, Hankins JV, et al. Periplasmic phosphorylation of lipid A is linked to the synthesis of undecaprenyl phosphate. Molecular Microbiology 2008;67:264.

19. Schumacher MA, Piro KM, Xu W, et al. Molecular mechanisms of HipA-mediated multidrug tolerance and its neutralization by HipB. Science 2009;323:396-401.

20. Chen HD, Groisman EA. The biology of the PmrA/PmrB two-component system: the major regulator of lipopolysaccharide modifications. Annu Rev Microbiol 2013;67:83-112.

21. Lee K, Yong D, Jeong SH, et al. Multidrug-resistant Acinetobacter spp.: increasingly problematic nosocomial pathogens. Yonsei Med J 2011;52:879-891.

22. Arroyo LA, Herrera CM, Fernandez $L$, et al. The pmrCAB Operon Mediates Polymyxin Resistance in Acinetobacter baumannii ATCC 17978 and Clinical Isolates through Phosphoethanolamine Modification of Lipid A. Antimicrobial Agents \& Chemotherapy 2011;55:3743.

23. Lorenz C, Dougherty TJ, Lory S. Transcriptional Responses of Escherichia coli to a Small-Molecule Inhibitor of LolCDE, an Essential Component of the Lipoprotein Transport Pathway. J Bacteriol 2016;198:3162-3175.

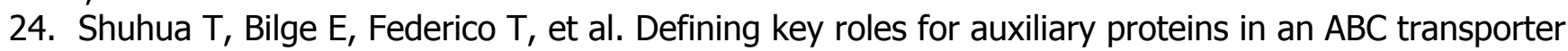
that maintains bacterial outer membrane lipid asymmetry. Elife 2016;5.

25. Simpson BW, Owens TW, Orabella MJ, et al. Identification of Residues in the Lipopolysaccharide ABC Transporter That Coordinate ATPase Activity with Extractor Function. mBio 2016;7.

26. Zaprasis A, Brill J, Thuring $M$, et al. Osmoprotection of Bacillus subtilis through import and proteolysis of proline-containing peptides. Applied and environmental microbiology 2013;79:576-587. 27. Weinberg MV, Maier RJ. Peptide transport in Helicobacter pylori: roles of dpp and opp systems and evidence for additional peptide transporters. J Bacteriol 2007;189:3392-3402. 


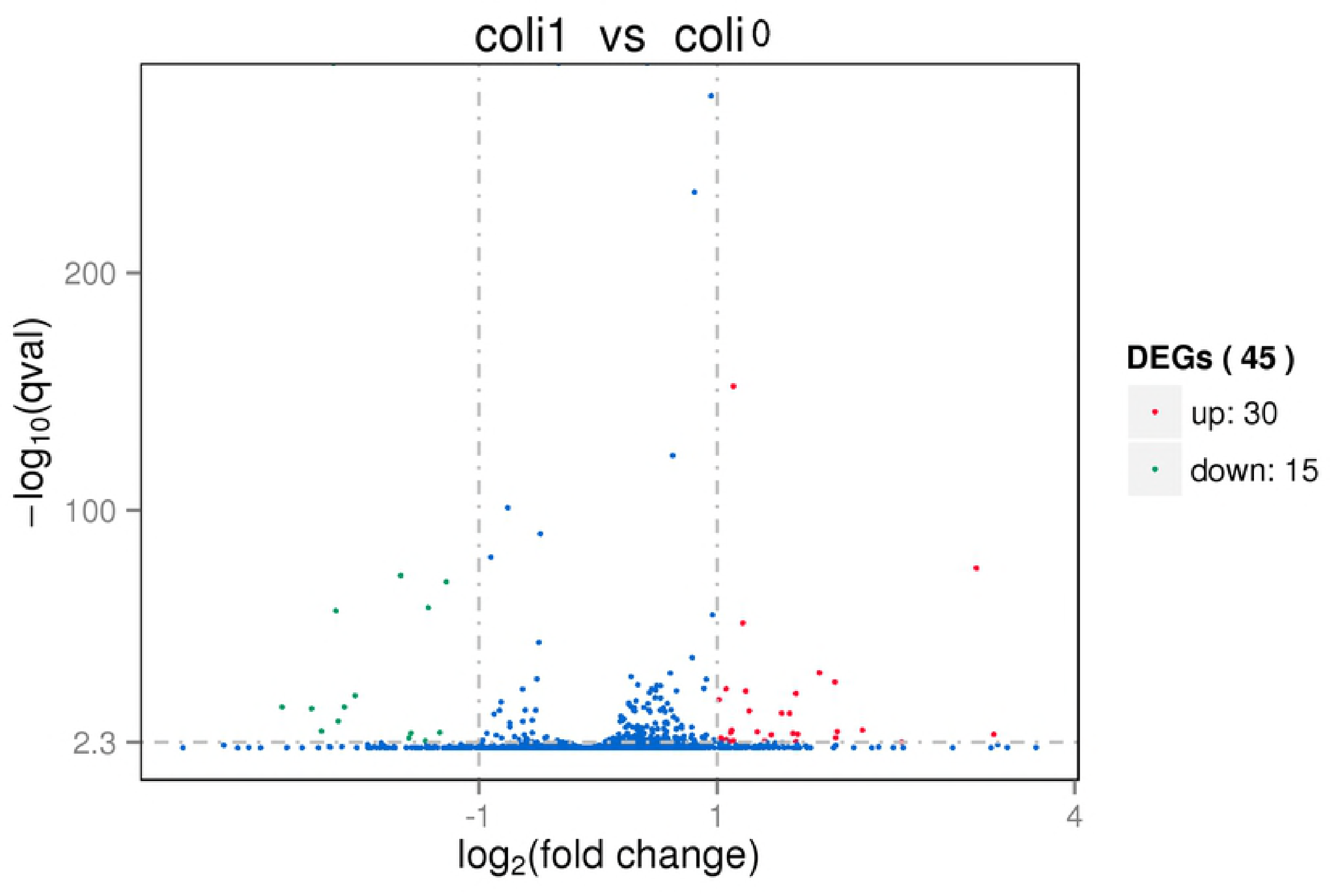





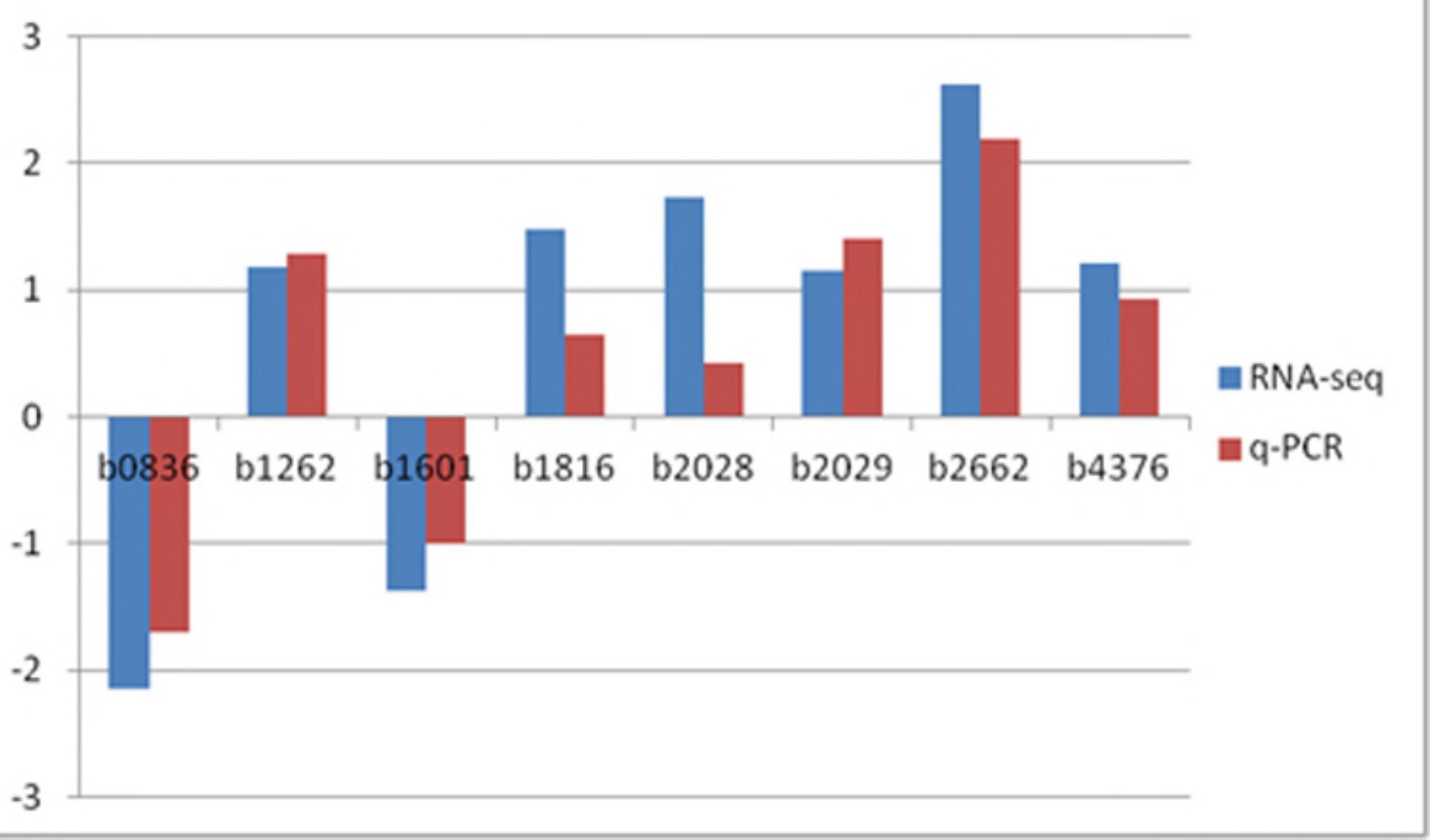

